\title{
La comunicación de los ayuntamientos en las redes sociales: participación ciudadana, información de servicio público y campaña permanente Municipalities' communication on social networks: citizen participation, public service information or permanent campaign
}

Laura Cervi Universitat Autònoma de Barcelona

Carles Marín-Lladó Universidad Rey Juan Carlos

Carmina Oliveras-Vila Universitat Autònoma de Barcelona

\section{Referencia de este artículo}

Cervi, Laura, Marín-Lladó, Carles y Oliveras-Vila, Carmina (2022). La comunicación de los ayuntamientos en las redes sociales: participación ciudadana, información de servicio público y campaña permanente. En: adComunica. Revista Científica de Estrategias, Tendencias e Innovación en Comunicación, $\mathrm{n}^{0} 23$. Castellón de la Plana: Departamento de Ciencias de la Comunicación de la Universitat Jaume I, 275-299. DOI: http://dx.doi.org/10.6035/adcomunica.6180.

\section{Palabras clave}

Redes sociales; ayuntamientos; comunicación política; participación ciudadana.

\section{Keywords}

Social Networks; Municipalities; Political Communication; Citizen Participation. 


\title{
Resumen
}

Este artículo explora el uso que hace la Administración pública local de las tres principales redes sociales (Twitter, Facebook e Instagram) con el objetivo de comprobar si los ayuntamientos aprovechan todas sus potencialidades, incentivan la participación ciudadana a partir de la comunicación de la actividad municipal, aumentan la calidad del servicio público, y ensalzan o no los logros de gobierno de sus líderes políticos. Todo ello, teniendo en cuenta los criterios básicos de la comunicación de las instituciones públicas y de la comunicación política, así como el concepto de campaña permanente. Para ello, se ha realizado un seguimiento de las redes sociales de los Ayuntamientos de Barcelona, Sabadell y Manresa, llevando a cabo un análisis cuantitativo y cualitativo en el que se han categorizado aspectos como las llamadas a la participación que hace la Administración, la temática de sus publicaciones, el apoyo o rechazo que consiguen a través de los likes y los comentarios, el contenido de la información (de servicio público, de actividad municipal y de contenido político), y la presencia o ausencia de los gobiernos y sus líderes en los mensajes emitidos. Los resultados evidencian que la Administración pública local no aprovecha las potencialidades que ofrecen las redes sociales para impulsar la participación ciudadana en los asuntos públicos. Aunque su implantación se encuentra aún en una fase inicial, este organismo público local ejerce una comunicación unidireccional, con información de servicio público, pero también con contenido político e ideológico.

\begin{abstract}
This article explores how local public administration uses three main social networks (Twitter, Facebook and Instagram) with the aim of verifying if the municipalities take advantage of all their potentialities, encourage citizen participation from the communication of municipal activity, increase the quality of public service, and praise or not the government achievements of their political leaders. All this, taking into account the basic criteria of the communication of public institutions and political communication, as well as the concept of permanent campaign. To this end, the social networks of the City Councils of Barcelona, Sabadell and Manresa have been monitored, carrying out a quantitative and qualitative analysis in which aspects such as the calls for participation made by the Administration, the subject $r$ of the posts, the support or rejection obtained through likes and comments, the content of the information (public service, municipal activity and political content), and the presence or absence of the governments and their leaders in the messages issued have been categorized. The results show that the local public administration does not take advantage of the potential offered by social networks to promote citizen participation in public affairs. Although its implementation is still in an initial phase, this local public body exercises a unidirectional communication, with public service information, but also with political and ideological content.
\end{abstract}




\section{Autores}

Laura Cervi [Laura.Cervi@uab.cat] es Profesora Serra Hunter e investigadora del Departamento de Periodismo y Ciencias de la Comunicación de la UAB. Doctora en Ciencia. Es miembro del grupo de investigación Gabinete de Comunicación y Educación y coordinadora académica del Máster en Gestión de la Comunicación Política y Electoral del Departamento de Periodismo y Ciencias de la Comunicación de la UAB.

Carles Marín-Lladó [carles.marin@urjc.es] es Profesor titular de Periodismo Audiovisual en la Universidad Rey Juan Carlos. Se ha especializado en la información televisiva y radiofónica a partir de su análisis discursivo. Ha publicado una decena de libros y numerosos artículos sobre reporterismo e informativos audiovisuales. Ha sido vicerrector y vicedecano de la URJC. Dirige el Máster en Reporterismo de Televisión en la misma universidad.

Carmina Oliveras-Vila [carmina.oliveras@e-campus.uab.cat] es licenciada en Periodismo por la UAB y Master Internacional de Gestión de la Comunicación Política y Electoral. Trabajó para los diarios Regió7 y Público entre 2007 y 2016 . Fue jefa de redacción de TLB Grupo Comunicación, donde puso en marcha el digital manresadiari.cat y el lanzamiento regional del diario El Punt Avui. 


\section{Introducción}

El siglo XXI ha consolidado definitivamente la Sociedad de la Información y la Comunicación en la que vivimos, después de que la tecnología, en todas sus vertientes y opciones, se haya instalado de forma definitiva en nuestras vidas.

En particular, la llamada Web Social o Web 2.0 y la aparición y asentamiento de las redes sociales han abierto un nuevo mundo que ha transformado nuestra vida cotidiana y la manera de comunicarnos (Papacharissi, 2010), a través de la interactuación de los usuarios y a partir de su capacidad de creación de contenidos.

Empresas e instituciones, tanto públicas como privadas, han tenido que adaptarse a esa forma de comunicación rápida e instantánea para llegar a los ciudadanos e interactuar con ellos (Baldwin-Philippi, 2015). Y en este sentido la política no se ha quedado atrás; la mayoría de sus actores son activos en las redes sociales (Dimitrova, Matthes, 2018; Casero-Ripollés, 2018; Cervi, 2020a) y, a través de ellas, perpetúan una campaña que ha pasado a convertirse en permanente. Así pues, los políticos y sus partidos necesitan comunicarse constantemente, tanto en las campañas como en los periodos rutinarios (Vasko; Trilling, 2019), y las Redes, a este respecto, se muestran como una alternativa más barata y rápida a las herramientas de los medios tradicionales. Además, la naturaleza desintermediada de los medios sociales ofrece la oportunidad de eludir a los gatekeepers, comunicándose directamente con los ciudadanos (Cervi, 2020b). Por esta razón, la mayoría de las instituciones dispone de cuentas - principalmente Facebook, Twitter e Instagram- que utiliza como una herramienta más dentro de sus dispositivos de gestión (Criado y Rojas, 2015) y que ayudan a agilizar la distribución de información a la ciudadanía (Criado, et al., 2017).

Las potencialidades de las redes sociales en la Administración son evidentes: consiguen no solo que la población participe en los asuntos públicos (Rivero-Hernández, 2017; Campillo-Alhama, 2010), sino que se les devuelva un cierto control sobre la agenda política (Chaves-Montero, 2017). Muchos autores constatan que, en manos de la Administración pública, las redes sociales pueden y deben incentivar el diálogo y el debate para llegar a mayores consensos ciudadanos, sobre todo en los ayuntamientos, considerados el primer eslabón de la atención ciudadana (Bertot, et al., 2012a; Bonsón-Ponte, et al., 2013; Criado y Rojas, 2012, 2015; Ellison y Hardey; 2014; Mickoleit, 2014; Song y Lee, 2016; Yi, Oh y Kim, 2013).

Por eso, en el terreno de la Administración pública se hace necesario diferenciar lo que es información de servicio público y de interés para el ciudadano de lo que es información de contenido político (Escalona-Nicolás, 2015). Pero no es tarea fácil. La línea que separa el ámbito de la comunicación de las instituciones públicas del ámbito de la comunicación política - vinculada a los partidos y a sus líderes- es, a menudo, movible (Canel-Crespo, 2018). Asimismo, existen puntos de encuentro entre los dos tipos de comunicación (Escalona-Nicolás, 2015), de tal manera que, 
en ocasiones, se hace difícil determinar hasta qué punto los mensajes emitidos por una institución pública tienen una dimensión netamente informativa y neutra o cuándo llevan implícitos contenidos ideológicos que buscan un rédito político (Canel-Crespo, 2018).

El artículo revisa la teoría existente en el campo de la comunicación de las instituciones públicas (Medaglia y Zheng, 2017), de la comunicación política, de las funciones de la Administración pública y de la propia implantación en las redes sociales, teniendo en cuenta también la legislación vigente y el escenario actual de desarrollo de las funciones, que ya es plenamente digital, y que permite la participación de la ciudadanía. Recogiendo la teoría expuesta, la investigación se centra en el estudio y la comparación de la comunicación que se emite desde las redes sociales (Facebook, Twitter e Instagram) de tres ayuntamientos, concebidos como el órgano administrativo más próximo a los ciudadanos. Se trata de los consistorios de Barcelona, Sabadell y Manresa, todos ellos ubicados en Cataluña, que cuentan con diferencias substanciales como, por ejemplo, el PIB o el número de habitantes.

Este trabajo tiene los objetivos siguientes: (1) Estudiar si se aprovechan todas las potencialidades que ofrecen las redes sociales; (2) comprobar si la comunicación que los ayuntamientos están ofreciendo a la ciudadanía se corresponde con la voluntad de incentivar la participación; (3) aumentar la calidad del servicio público e informar de la actividad municipal; y (4) observar si ésta se corresponde o no con una voluntad de promoción política que ensalza los logros del gobierno y sitúa al alcalde o alcaldesa como centro de atención.

\section{Marco}

\subsection{La comunicación de las instituciones públicas: obligaciones y límites}

Dentro de la Administración pública local coinciden dos ramas de la comunicación: la del día a día de este servicio público y la que hace referencia a la vertiente política de un líder (o de un gobierno) que quiere continuar en el poder.

Campillo-Alhama (2010) sostiene que, a través de diversos procesos comunicativos y relacionales, «las administraciones se dirigen a la ciudadanía para transmitir todo tipo de mensajes relacionados con el desarrollo de competencias públicas, unos procesos que se justifican desde la premisa fundamental de que el ciudadano, como epicentro de la acción de gobierno local, debe estar informado de todas aquellas actuaciones e iniciativas administrativas que pueden incidir en su condición de ciudadano-administrado».

En cuanto al objeto de los mensajes, el elemento de la participación es visto como fundamental en una comunicación que, dentro de un contexto democrático, también debe perseguir el objetivo de ser un instrumento eficaz para asegurar la 
participación de los ciudadanos en los asuntos públicos y hacer que se sientan responsables de sus elecciones (Campillo-Alhama, 2010). Asimismo, y para diferenciar la comunicación de las instituciones públicas de la comunicación política y electoral, el objetivo de estos mensajes debe ser solamente la difusión de la actividad de la entidad como tal, y no de los miembros que lo configuran, con un contenido que debe transmitir información objetiva y neutra que se adecúe a su vocación irrenunciable de servicio público, huyendo del discurso del partido político y de la contienda electoral (Aira-Foix et al., 2019) que provoca que, desde muchas administraciones públicas, se esté en campaña permanente.

\subsection{La regulación estatal}

En España, la Ley 29/2005, de 29 de diciembre, de Publicidad y Comunicación Institucional (BOE, 2005), regula la comunicación y la publicidad promovidas o contratadas por la Administración General del Estado y por las demás entidades integrantes del sector público estatal, y establece las pautas para su diferenciación con respecto a la comunicación política. En el artículo número 4 de esta Ley se encuentra la regulación de las prohibiciones que, básicamente, impide a los gobiernos promover o contratar campañas institucionales de publicidad y de comunicación que tengan como finalidad destacar los logros de gestión o los objetivos alcanzados. Además, en los períodos electorales la ley se endurece. Están específicamente regulados en su artículo 10 y prohíben cualquier tipo campaña institucional durante este tiempo, sea cual sea su objeto. Cuando la norma se aprobó, el entonces presidente José Luís Rodríguez Zapatero (PSOE) aseguró que la Ley permitiría acabar con las campañas de «autobombo y favoritismo» que desde la Administración pública hacían de forma recurrente algunos partidos.

\subsection{Administración municipal, participación y redes sociales}

El cometido fundamental de la Administración municipal consiste en garantizar el interés público a través del desarrollo de las competencias o funciones que tiene asignadas (Campillo-Alhama, 2010), como satisfacer las necesidades de una sociedad por medio de los servicios públicos (Canel-Crespo, 2018). El ayuntamiento es el órgano administrativo de menor rango territorial y el más próximo a los ciudadanos, y estos deben ser llamados a participar de la vida colectiva y política generada por la propia administración con el fin de asegurar la construcción de comunidad y el interés público (Rivero-Hernández, 2017).

De la aludida Web Social se derivan las redes sociales, que abren la posibilidad de interacción entre usuarios y también la posibilidad de aumentar la relación entre Administración y ciudadano. Para la Administración pública, representan una gran oportunidad para asegurar, promover e instaurar la deseada participación de la 
ciudadanía en los asuntos públicos, y para devolverles también la agenda política (Chaves-Montero, 2017), y aunque la tecnología está de su parte, no parece que se haya avanzado mucho en este campo (Criado y Rojas, 2015; Criado, Pastor y Villodre, 2018).

En general, la literatura sugiere que las redes sociales, aplicadas a las administraciones, pueden ayudar a transformar las organizaciones públicas, haciéndolas más abiertas, permeables y cercanas a los ciudadanos (Song y Lee, 2015; Bertot et al., 2012b). Diferentes autores consideran incluso que las redes sociales contribuyen a crear información pública de forma colectiva (Graham y Avery, 2013), así como a aumentar la percepción de la transparencia por parte de la ciudadanía (Song y Lee, 2015; Meijer y Thaens, 2013; Bertot et al., 2012a; Bonsón-Ponte et al., 2013), generando confianza en las instituciones públicas e impulsando la participación (Mickoleit, 2014; Ellison y Hardey, 2014; Bertot et al., 2012b). También contribuyen a la coproducción de materiales y servicios públicos (Mickoleit, 2014; Bertot et al., 2012b), y a la idea del gobierno abierto (Bertot et al., 2012a).

Sin embargo, según otros autores (Criado y Rojas-Martín, 2013; Graells y Ramilo, 2013; Mickoleit, 2014; Bonsón-Ponte et al. 2013; Agostino, 2013; Mossberger, Wu y Crawford, 2013; Ellison y Hardey, 2014) no hay evidencias que constaten que estas tecnologías estén logrando esos objetivos (Criado, 2013; Gascó, 2014), puesto que los potenciales beneficios de estas técnicas sociales están siendo infrautilizados actualmente (Graham y Avery, 2013).

\section{Objetivos y metodología}

Este trabajo parte de unos objetivos que justifican el uso que hace la Administración pública local de las redes sociales más importantes para su ámbito de actuación (Twitter, Facebook e Instagram), que son: observar las potencialidades de estos medios sociales; comprobar si los ayuntamientos ofrecen a la ciudadanía una incentivada voluntad para que participe de sus logros; informar de la actividad municipal y del aumento de la calidad en el servicio público; y observar si existe una promoción política de ensalzamiento de lo realizado por el gobierno y por sus alcaldes, como máximos responsables. Para ello, este estudio se basa en el análisis y comparación de tres casos concretos: el Ayuntamiento de Barcelona, el Ayuntamiento de Sabadell y el Ayuntamiento de Manresa. Se escogen tres ciudades dentro de una misma Comunidad Autónoma por motivos geopolíticos y de proximidad: Cataluña -con sus diferencias en el número y tipología de población-, con la finalidad de comprobar si el uso de las redes sociales de los consistorios ejerce una influencia o no en sus conciudadanos. 
Tabla 1. Datos demográficos y socioeconómicos de los tres municipios

\begin{tabular}{|l|c|c|c|c|} 
Ciudad & Habitantes & $\begin{array}{c}\text { \% Población nacionalidad } \\
\text { extranjera }\end{array}$ & $\begin{array}{c}\text { PIB (millones de } \\
\text { euros) }\end{array}$ & $\begin{array}{c}\text { PIB por habitante } \\
\text { (millones de euros) }\end{array}$ \\
\hline Barcelona & 1.636 .762 & 19,58 & $72.257,2$ & 45 \\
\hline Sabadell & 213.644 & 12,04 & $4.454,9$ & 21,2 \\
\hline Manresa & 77.714 & 17,65 & $1.929,2$ & 25,7 \\
\hline
\end{tabular}

Fuente: Idescat, 2019

En concreto, el análisis se centra en Facebook (en adelante FB), Instagram (en adelante IG) y Twitter (en adelante TW), tres de las cinco redes sociales con más seguimiento en España. Se ha descartado Whatsapp al ser una red social de ámbito y uso personal, y Youtube por la escasa o nula actividad por parte de los ayuntamientos analizados. El corpus del estudio se compone de 356 tuits, 105 publicaciones en Facebook y 37 publicaciones en Instagram. El artículo queda delimitado en los perfiles corporativos principales (FB, TW e IG) de las tres administraciones locales, que son los que el usuario irá a buscar en primera instancia cuando necesite información o quiera interactuar con su ayuntamiento. Y dentro de ellos, queda limitado a las publicaciones (posts) que se encuentran en la cronología (timeline). Siguiendo las principales teorías recogidas en el apartado del marco teórico, así como los retos que en él se plantean y los estudios previos en el ámbito español (Criado, Villodre. 2018; Fernández-Falero, et alt., 2017; Zavattaro, French y Mohanty, 2015), este trabajo aborda las siguientes preguntas de investigación:

a. ¿Contribuyen los ayuntamientos a impulsar la participación de la ciudadanía en los asuntos públicos a través de las redes sociales?

b. ¿Su comunicación es unidireccional, bidireccional o multidireccional?

c. ¿Promueven el diálogo entre la ciudadanía y entre la Administración y la ciudadanía?

d. ¿Se difunde la actividad del consistorio o la actividad de los miembros del Gobierno?

e. ¿Con qué frecuencia aparecen los miembros del Gobierno?

f. ¿La información contiene contenido político?

g. ¿Qué tipo de comunicación predomina: la comunicación política o la comunicación de las instituciones públicas? 
La investigación se centra en los mensajes emitidos durante dos semanas y en los que cada ayuntamiento habrá celebrado la sesión del pleno municipal ordinario del mes de octubre de 2020. Se opta por introducir la celebración de un pleno dentro del periodo estudiado por ser el órgano colegiado -formado por el alcalde o alcaldesa y todos los concejales de gobierno y oposición- que decide y aprueba las principales políticas que afectan a la ciudad y a sus ciudadanos. Así, la investigación de campo queda delimitada en estos tres períodos:

- Ayuntamiento de Barcelona: del 26 de octubre al 8 de noviembre. Celebración del pleno: 30 de octubre.

- Ayuntamiento de Sabadell: del 28 de septiembre al 11 de octubre. Celebración el pleno: 6 de octubre.

- Ayuntamiento de Manresa: del 5 de octubre al 18 de octubre. Celebración del pleno: 15 de octubre.

Este estudio combina análisis de contenido cuantitativo y cualitativo. La unidad de análisis son los posts, cuya materia ha sido cifrada por dos codificadores, basándose en una hoja de cálculo construida sobre una variedad de indicadores, incluyendo criterios formales y características relacionadas con el contenido.

El marco de codificación consiste en una serie de preguntas dicotómicas con respuesta sí/no, a las que se asignaron valores empíricos de 1/0 para permitir el tratamiento de los datos por el SPSS. Este método, basado en una propuesta de Vergeer, Hermans y Cunha (2013) y aplicado, entre otros, por Cervi y Roca (2017a, 2017b), lo que permite la medición de cada categoría de forma empírica y, por tanto, su comparación.

En los tres casos se analizan los siguientes parámetros:

- Número de publicaciones totales y diarias

- Número de reproducciones de vídeo (RV) en las publicaciones que lo contienen

- Números de likes (LK) *En Facebook se incluyen las reacciones positivas

- Número de reacciones negativas (R-) *Solo en Facebook

- Número de comparticiones o retuits (CP)

- Número de comentarios (CO)

- Número de comentarios positivos $(\mathrm{CO}+)^{*}$ De aprobación; incluye las preguntas que el usuario hace a la Administración 
- Número de comentarios negativos (CO-) *De rechazo

- Llamada a la participación por parte de la Administración (LLP)

- Respuestas de la Administración dentro del post (RA)

- Información de servicio público (ISP)

- Información sobre la actividad municipal que desarrolla el Ayuntamiento (IAM)

- Presencia del alcalde o la alcaldesa (PA)

- Presencia del resto del gobierno (PG)

- Logros del Gobierno (LG)

- Contenido político/ideológico (CP)

\section{Resultados}

\subsection{Las redes sociales de la Administración pública local}

Los usuarios de Internet en general, y de las redes sociales en particular, no han parado de crecer en los últimos años. El Instituto Nacional de Estadística (INE) señala que, en el último trimestre de 2019, el 90,7\% de la población española de 16 a 74 años utilizó Internet (2020).

En lo referente a las redes sociales, su uso global en 2019 ha aumentado más del $9 \%$ con respecto al año anterior, acumulando ya más de 3.800 millones de usuarios activos en todo el mundo, según datos del Informe Digital 2020, elaborado por Hootsuite y We Are Social. En cuanto a España, el Estudio Anual de Redes Sociales 2019, presentado por IAB (2019) (la asociación mundial de comunicación, publicidad y marketing digital) indica que el 85,5\% de los internautas de 16 a 65 años utiliza redes sociales, lo que representa más de 25 millones usuarios en España. El mismo informe constata que la red social más usada es WhatsApp (88\%), seguida de Facebook (FB) (87\%), YouTube (68\%), Instagram (IG) (54\%) y Twitter (TW), con un 50\% (Figura 1).

En lo referente a la Administración pública local, el estudio de Criado, Pastor y Villodre (2018) indica que el 84,9\% de los ayuntamientos utiliza las redes sociales como una herramienta más dentro de sus dispositivos de gestión. De entre las principales plataformas digitales disponibles (Figura 2), prácticamente la totalidad están utilizando de manera prioritaria TW $(99,2 \%)$ y FB $(96,7 \%)$, y en menor medida YouTube (79,3\%). En este estudio, de 2018, IG aparece en sexta posición $(36,4 \%)$, si bien hay una tendencia al alza de esta red social dentro de la Administración pública local. 
Figura 1. Uso de redes sociales en España

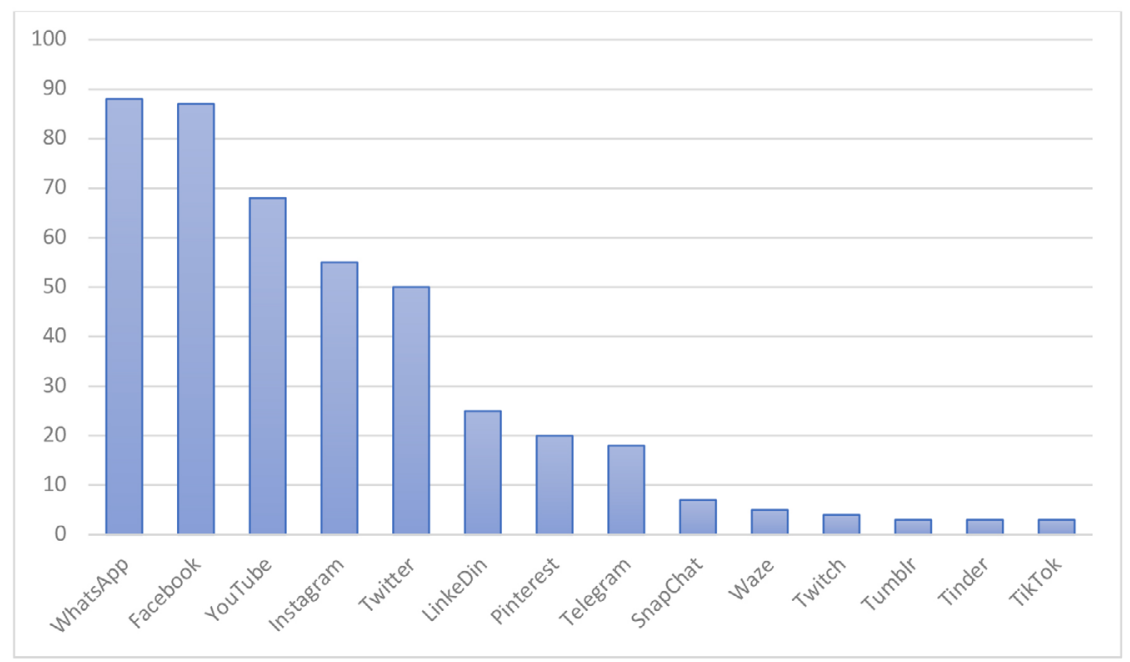

Fuente: Iab/Adglow/Elogia/Epdata.es (2019)

Figura 2. Presencia de plataformas digitales en los Ayuntamientos de más de 50.000 habitantes.

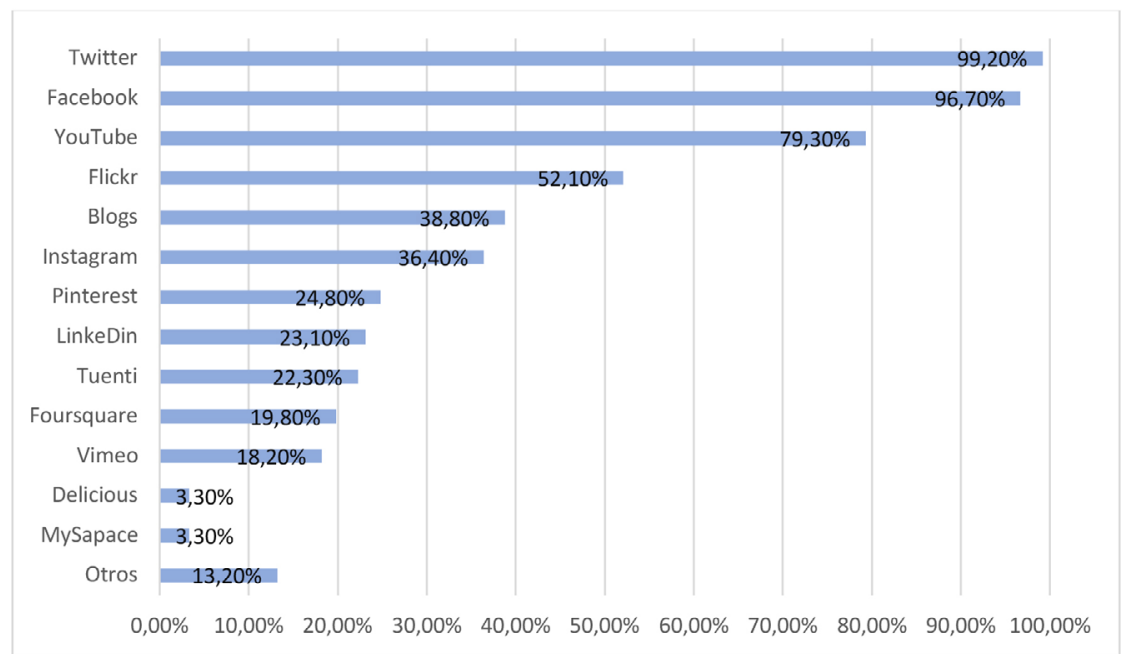

Fuente: Criado, Pastor y Villodre (2018) 
El análisis de los perfiles de los Ayuntamientos de Barcelona, Sabadell y Manresa sigue la tendencia de la Administración pública local española. La primera red social que abrieron fue TW. Barcelona lo hizo en diciembre de 2009, Sabadell en septiembre de 2010, y Manresa en diciembre de 2010. Esta red es la que cuenta con más seguidores en Barcelona, con bastante diferencia con respecto a FB e IG, que siguen un orden de adeptos de acuerdo con la fecha de apertura: cuanto más antigua es la red, más seguidores acumula. No sucede así en Sabadell y Manresa, donde ya se visualiza el fenómeno IG. En Sabadell, IG ya es la red con más seguidores - aunque es la más joven-, pasando por poco a TW y a mucha diferencia con respecto a FB. En Manresa, sigue la misma tendencia: pese a que TW sigue siendo la red con más seguidores, tiene ya muy cerca a IG, que ha sobrepasado a FB y está a punto de alcanzar a TW.

Tabla 2. Apertura, seguidores y penetración

\begin{tabular}{|l|c|c|c|c|}
\hline Ciudad & Red social & Creada en & Seguidores & Penetración* \\
\cline { 2 - 5 } & Twitter & Dic-09 & 414.310 & $25,31 \%$ \\
\cline { 2 - 5 } & Facebook & Sept-10 & 237.960 & $14,54 \%$ \\
\cline { 2 - 5 } & Instagram & Feb-12 & 181.000 & $11,05 \%$ \\
\hline \multirow{3}{*}{ Sabadell } & Twitter & Sept-10 & 13.393 & $6,27 \%$ \\
\cline { 2 - 5 } & Facebook & Nov-11 & 5.130 & $2,40 \%$ \\
\cline { 2 - 5 } & Instagram & Feb-14 & 15.000 & $7,02 \%$ \\
\hline \multirow{3}{*}{ Manresa } & Twitter & Dic-10 & 9.230 & $11,88 \%$ \\
\cline { 2 - 5 } & Facebook & Abr-11 & 6.568 & $8,45 \%$ \\
\cline { 2 - 5 } & Instagram & Des-14 & 8.883 & $11,43 \%$ \\
\hline
\end{tabular}

*Porcentaje de usuarios con respecto a los habitantes

Fuente: elaboración propia, a fecha 8/11/2020.

\subsection{Conceptos analizados}

En las siguientes tablas se muestra el recuento de los conceptos analizados ${ }^{1}$, $\operatorname{co}^{-}$ rrespondientes al vaciado de datos del Ayuntamiento de Barcelona, realizado entre el 26 de octubre y el 8 de noviembre de 2020 (Tabla 3); del Ayuntamiento de Sabadell, realizado entre el 28 de septiembre y el 11 de octubre de 2020 (Tabla 4); y del Ayuntamiento de Manresa, realizado entre el 5 y el 18 de octubre de 2020

1 Si bien se contabilizan, entre otras nociones, las interacciones en una red social, por ejemplo: los likes, como forma de participación, también se computan otras como el hecho de compartir un contenido publicado, sin que haya necesariamente una reacción positiva, negativa e incluso neutra del usuario. 
(Tabla 5). Seguidamente (Tabla 6), se muestra qué red social es la más activa por cada concepto analizado.

Tabla 3. Datos Ayuntamiento de Barcelona

\begin{tabular}{|c|c|c|l|}
\hline TW & FB & IG & Conceptos analizados \\
\hline 172 & 15 & 13 & Número de publicaciones totales \\
\hline 12 & 1 & 0,9 & Media de publicaciones al día \\
\hline 7.309 & 2.290 & 5.762 & Media de reproducciones en las publicaciones con vídeo \\
\hline 4 & 66 & 739 & Media de likes en cada publicación* \\
\hline- & 9 & - & Media de reacciones negativas en cada publicación** \\
\hline- & $88 \%$ & - & Porcentaje de likes y reacciones positivas** \\
\hline- & $12 \%$ & - & Porcentaje de reacciones negativas** \\
\hline 2 & 12 & - & Media de comparticiones en cada publicación o RT \\
\hline 0,1 & 19 & 15 & Media de comentarios en cada publicación \\
\hline $74 \%$ & $93 \%$ & $39 \%$ & Porcentaje de comentarios negativos \\
\hline $26 \%$ & $7 \%$ & $61 \%$ & Porcentaje de comentarios positivos \\
\hline $11 \%$ & $7 \%$ & $7 \%$ & Publicaciones que llaman a la participación \\
\hline $0 \%$ & $0 \%$ & $0,2 \%$ & Respuestas de la Administración \\
\hline $56 \%$ & $73 \%$ & $60 \%$ & Publicaciones que contienen información de servicio público \\
\hline $15 \%$ & $47 \%$ & $38 \%$ & Publicaciones que contienen información de la actividad municipal que se desarrolla \\
\hline $0 \%$ & $0 \%$ & $0 \%$ & Publicaciones en las que se cita o aparece el/la alcalde/esa \\
\hline $0 \%$ & $0 \%$ & $0 \%$ & Publicaciones en las que se cita o aparecen el resto de los miembros del gobierno \\
\hline $6 \%$ & $20 \%$ & $15 \%$ & Publicaciones en las que se informa de logros del gobierno \\
\hline $20 \%$ & $33 \%$ & $31 \%$ & Publicaciones que contienen información de contenido político/ideológico \\
\hline No & No & No & Se informa del pleno municipal \\
\hline
\end{tabular}

${ }^{*}$ En Facebook se contabilizan también las reacciones positivas

**Sólo en Facebook

Fuente: TW, FB e IG del Ayuntamiento de Barcelona. Elaboración propia. 
Tabla 4. Datos Ayuntamiento de Sabadell

\begin{tabular}{|c|c|c|c|}
\hline TW & FB & IG & Conceptos analizados \\
\hline 108 & 51 & 9 & Número de publicaciones totales \\
\hline 8 & 4 & 0,6 & Media de publicaciones al día \\
\hline 332 & 480 & 2.150 & Media de reproducciones en las publicaciones con vídeo \\
\hline 6 & 11 & 151 & Media de likes en cada publicación* \\
\hline- & 4 & - & Media de reacciones negativas en cada publicación** \\
\hline- & $76 \%$ & - & Porcentaje de likes y reacciones positivas** \\
\hline- & $24 \%$ & - & Porcentaje de reacciones negativas** \\
\hline 4 & 6 & - & Media de comparticiones en cada publicación o RT \\
\hline 0,3 & 4 & 5 & Media de comentarios en cada publicación \\
\hline $41 \%$ & $97 \%$ & $77 \%$ & Porcentaje de comentarios negativos \\
\hline $59 \%$ & $3 \%$ & $11 \%$ & Porcentaje de comentarios positivos \\
\hline $0 \%$ & $0 \%$ & $0 \%$ & Publicaciones que llaman a la participación \\
\hline $0 \%$ & $0 \%$ & $0 \%$ & Respuestas de la Administración \\
\hline $65 \%$ & $71 \%$ & $67 \%$ & Publicaciones que contienen información de servicio público \\
\hline $40 \%$ & $18 \%$ & $11 \%$ & Publicaciones que contienen información de la actividad municipal que se desarrolla \\
\hline $17 \%$ & $12 \%$ & $22 \%$ & Publicaciones en las que se cita o aparece el/la alcalde/esa \\
\hline $29 \%$ & $14 \%$ & $22 \%$ & Publicaciones en las que se cita o aparecen el resto de los miembros del gobierno \\
\hline $12 \%$ & $6 \%$ & $11 \%$ & Publicaciones en las que se informa de logros del gobierno \\
\hline $31 \%$ & $22 \%$ & $33 \%$ & Publicaciones que contienen información de contenido político/ideológico \\
\hline Sí & No & No & Se informa del pleno municipal \\
\hline
\end{tabular}

${ }^{*}$ En Facebook se contabilizan también las reacciones positivas

**Sólo en Facebook

Fuente: TW, FB e IG del Ayuntamiento de Sabadell. Elaboración propia. 
Tabla 5. Datos Ayuntamiento de Manresa

\begin{tabular}{|c|c|c|l|}
\hline TW & FB & IG & Conceptos analizados \\
\hline 76 & 39 & 15 & Número de publicaciones totales \\
\hline 5 & 3 & 1 & Media de publicaciones al día \\
\hline 292 & 149 & 1.318 & Media de reproducciones en las publicaciones con vídeo \\
\hline 7 & 16 & 208 & Media de likes en cada publicación* \\
\hline- & 0,3 & - & Media de reacciones negativas en cada publicación** \\
\hline- & $98 \%$ & - & Porcentaje de likes y reacciones positivas** \\
\hline- & $2 \%$ & - & Porcentaje de reacciones negativas** \\
\hline 3 & 5 & - & Media de comparticiones en cada publicación o RT \\
\hline 0,25 & 1 & 2 & Media de comentarios en cada publicación \\
\hline $53 \%$ & $76 \%$ & $42 \%$ & Porcentaje de comentarios negativos \\
\hline $47 \%$ & $24 \%$ & $58 \%$ & Porcentaje de comentarios positivos \\
\hline $1 \%$ & $3 \%$ & $7 \%$ & Publicaciones que llaman a la participación \\
\hline $0 \%$ & $0 \%$ & $0 \%$ & Respuestas de la Administración \\
\hline $74 \%$ & $67 \%$ & $60 \%$ & Publicaciones que contienen información de servicio público \\
\hline $46 \%$ & $41 \%$ & $53 \%$ & Publicaciones que contienen información de la actividad municipal que se desarrolla \\
\hline $21 \%$ & $21 \%$ & $13 \%$ & Publicaciones en las que se cita o aparece el/la alcalde/esa \\
\hline $17 \%$ & $23 \%$ & $27 \%$ & Publicaciones en las que se cita o aparecen el resto de los miembros del gobierno \\
\hline $3 \%$ & $5 \%$ & $13 \%$ & Publicaciones en las que se informa de logros del gobierno \\
\hline $14 \%$ & $15 \%$ & $27 \%$ & Publicaciones que contienen información de contenido político/ideológico \\
\hline Sí & Sí & No & Se informa del pleno municipal \\
\hline
\end{tabular}

${ }^{*}$ En Facebook se contabilizan también las reacciones positivas

**Sólo en Facebook

Fuente: TW, FB e IG del Ayuntamiento de Manresa. Elaboración propia. 
Tabla 6. Redes sociales más activas por concepto analizado

\begin{tabular}{|l|c|c|c|}
\hline Concepto & Barcelona & Sabadell & Manresa \\
\hline Red en la que más publica el Ayuntamiento & Twitter & Twitter & Twitter \\
\hline Red que más reproducciones de vídeo obtiene & Twitter & Instagram & Instagram \\
\hline Red que más likes obtiene & Instagram & Instagram & Instagram \\
\hline Red que más comparticiones obtiene* & Facebook & Facebook & Facebook \\
\hline Red que más comentarios obtiene & Facebook & Instagram & Instagram \\
\hline $\begin{array}{l}\text { Red que, porcentualmente, más comentarios negativos } \\
\text { obtiene }\end{array}$ & Facebook & Instagram & Facebook \\
\hline Red que, porcentualmente, más comentarios positivos obtiene & Instagram & Twitter & Instagram \\
\hline $\begin{array}{l}\text { Red que, porcentualmente, da más información de servicio } \\
\text { público }\end{array}$ & Facebook & Facebook & Twitter \\
\hline $\begin{array}{l}\text { Red que, porcentualmente, da más información de la actividad } \\
\text { municipal }\end{array}$ & Facebook & Twitter & Instagram \\
\hline $\begin{array}{l}\text { Red en la que, porcentualmente, más aparece el/la alcalde/ } \\
\text { esa }\end{array}$ & Ninguna & Twitter & Twitter \\
\hline $\begin{array}{l}\text { Red en la que, porcentualmente, más aparece el resto del } \\
\text { gobierno }\end{array}$ & Ninguna & Twitter & Instagram \\
\hline $\begin{array}{l}\text { Red en la que, porcentualmente, más se informa de los logros } \\
\text { del gobierno }\end{array}$ & Facebook & Twitter & Instagram \\
\hline $\begin{array}{l}\text { Red que, porcentualmente, tiene más contenido político/ } \\
\text { ideológico }\end{array}$ & Facebook & Instagram & Instagram \\
\hline
\end{tabular}

*Sin contar Instagram, que no proporciona esta información

Fuente: Elaboración propia

\subsection{Uso de las redes sociales}

TW es la red más veterana en los tres ayuntamientos y es la que obtiene el mayor número de publicaciones. Sin embargo, se observa la tendencia al alza de IG entre los usuarios. Esta última es la red en la que los tres ayuntamientos obtienen más likes y, en el caso de Sabadell y Manresa, donde más reproducciones de vídeo recogen. Y, junto a FB, es donde se consiguen más comentarios de los ciudadanos, siendo los negativos el porcentaje mayoritario en los tres ayuntamientos; en IG, en cambio, se da el caso contrario. Se observa también que FB resiste como una de las principales redes en Barcelona, pero se va difuminando en las otras dos ciudades de menor población.

Así, el análisis indica que, aunque los usuarios comienzan a cambiarse a IG, los ayuntamientos todavía insisten en tener TW como red prioritaria para proporcionar información a la población. Aunque el formato de TW permite más publi- 
caciones que el de FB e IG, la diferencia entre el número de publicaciones de las tres redes es significativa. Y más si se tiene en cuenta la incidencia de estos tuits entre la ciudadanía.

En Barcelona, es muy baja, con solo 4 likes de media, por los 66 que consigue FB y los 739 de IG que, siendo la red con menos seguidores y la más joven, es la que aglutina ahora mismo más atención de la ciudadanía.

En Sabadell, IG suma 151 likes, en contraposición a los 11 de FB y los 6 de TW. La media de visualizaciones de IG en las publicaciones que contienen vídeo es de 2.150, mientras que FB tiene 480 y TW, 332.

En el caso de Manresa, también gana IG, con una media de 208 likes, mientras que FB obtiene 16 y TW, 7. En cuanto a visualizaciones de vídeo, IG tiene una media de 1.318, a diferencia de las 149 de FB y las 292 de TW, pero es la red en la que el Ayuntamiento publica menos temas.

\subsection{Participación}

Las tres administraciones locales practican una comunicación unidireccional y no aprovechan la posibilidad que ofrecen las redes sociales de abrir debates con la ciudadanía. A pesar de tener peticiones por parte de los ciudadanos, el Ayuntamiento de Manresa y el de Sabadell no responden en sus redes. El mismo camino sigue el de Barcelona: en TW y FB no responde en ninguna ocasión y, en el caso IG, es anecdótico: solo una contestación de 13 publicaciones en los 14 días analizados.

Hay que señalar también que la unidireccionalidad se acentúa más aún en el TW del Ayuntamiento de Barcelona, donde los mensajes (tuits) se programan y se lanzan a través de una plataforma de gestión de redes sociales (Hootsuite). A pesar de que existen maneras de monitorización y seguimiento a tiempo real, en los casos analizados no hemos observado que haya habido una interactuación, esto eso, una contestación al ciudadano por parte del consistorio, lo que no quiere decir que no se hayan producido en otros casos fuera de este análisis.

\subsection{Contenido de las publicaciones}

En cuanto al contenido de las publicaciones de los tres ayuntamientos, la mayoría incluye información de servicio público (Barcelona: 56\% en TW, 73\% en FB y 60\% en IG; Sabadell: 65\% en TW, 71\% en FB y 67\% en IG; Manresa: 74\% en TW, 67\% en FB y $60 \%$ en IG) y, en menores porcentajes, información sobre la actividad municipal que desarrolla cada Ayuntamiento (Barcelona 15\% en TW, 47\% en FB y $38 \%$ en IG; Sabadell: $40 \%$ en TW, $18 \%$ en FB y $11 \%$ en IG; Manresa: 46\% en TW, $41 \%$ en FB y $53 \%$ en IG).

Por otro lado, cuando nos fijamos en las veces que se cita el alcalde, la alcaldesa o sus equipos de gobierno desde los perfiles institucionales, es el Ayuntamiento de 
Barcelona el que da menos protagonismo a sus dirigentes: en ninguna de las tres redes sociales se cita a la alcaldesa ni a los concejales. No sucede así en Sabadell y en Manresa: citan o aparecen, en porcentajes idénticos, la alcaldesa y el alcalde en un $17 \%$ de las publicaciones, mientras que sus equipos de gobierno aparecen en un $22 \%$.

Por último, en el contenido político y/o ideológico de las publicaciones, se observa que se da en los tres ayuntamientos (Barcelona: 20\% en TW, 33\% en FB y $31 \%$ en IG; Sabadell: $31 \%$ en TW, 22\% en FB y 33\% en IG; Manresa: 14\% en TW, $15 \%$ en FB y 27\% en IG). En los tres consistorios también hay informaciones en las que se ensalzan los logros del gobierno (Barcelona: 6\% en TW, 20\% en FB y 15\% en IG; Sabadell: 12\% en TW, 6\% en FB y 11\% en IG; Manresa: 3\% en TW, $5 \%$ en FB y $13 \%$ en IG). En estos dos conceptos, el ayuntamiento más pequeño, el de Manresa, es el que menos lo hace (19\% de media de contenido político y $7 \%$ de media de logros del gobierno), mientras que el más grande, el de Barcelona, es el que publica más información política y de corte ideológico (29\%, en este caso empatado con Sabadell) y el que subraya más logros del gobierno (14\%), a pesar de que no salir políticos en sus publicaciones.

\section{Conclusiones}

\subsection{La participación ciudadana: tarea pendiente}

El análisis llevado a cabo en las tres principales redes sociales de los Ayuntamientos analizados permite afirmar que la "participación” es una tarea claramente pendiente. Ninguna aprovecha las potencialidades que ofrecen las redes sociales para impulsar la participación ciudadana en los asuntos públicos (Mickoleit, 2014; Ellison y Hardey, 2014; Bertot et al., 2012b) y las tres practican una comunicación 100\% unidireccional. Así, el debate entre Administración y ciudadano es nulo, un hecho que es totalmente contradictorio, si tenemos en cuenta que estas tecnologías se crearon, precisamente, para permitir la conexión, interacción e intercambio de información entre colectivos de personas que comparten intereses comunes (Boyd y Ellison, 2007; Chun et al., 2010)². Costaría encontrar un colectivo en el que se compartan más intereses comunes que el que conforman ciudadanía y ayuntamiento, organismo que, como se ha señalado, representa el primer eslabón de la atención ciudadana.

En 2020, la mayoría de los ayuntamientos ya había dado el salto a las redes sociales más populares, pero no parecía que hubieran conseguido explotarlas en toda su

2 No obstante, es interesante remarcar aquí que este trabajo hace referencia siempre a las cuentas principales de los ayuntamientos, puesto que estos pueden contener otras de departamentos específicos, por ejemplo: de participación ciudadana, de juventud o de cultura, donde la estrategia llevada a cabo puede ser diferente a la cuenta de referencia o principal. 
plenitud. Los resultados del presente trabajo ponen de manifiesto, como apuntaba ya el estudio de Criado y Rojas de 2015, que existe, por parte de los Ayuntamientos de Barcelona, Sabadell y Manresa, un amplio margen de mejora en el desarrollo de sus redes sociales y que, pese a la difusión que tienen en la actualidad -con un número significativo de seguidores y de publicaciones realizadas al día-, se encuentran en una fase de implantación inicial, ya que se está usando la misma comunicación unidireccional con la que se envía una cuña a la radio o se emite una nota de prensa, es decir, sin esperar ninguna retroactividad de la ciudadanía. Los tres ayuntamientos, por lo tanto, no promueven la participación e interacción; el uso que hacen de las redes sociales es meramente informativo, aunque sí ejercen una labor de prestación de servicio público propio de este tipo de administraciones (Criado y Villodre, 2021). No obstante, todavía queda lejos de implantarse otra manera interesante de aproximación por parte de la administración al ciudadano a través de las redes, por ejemplo: como ciudadano crítico o, simplemente, como cliente de su institución (Wukich, 2021).

Cabe señalar también que los resultados de la investigación de Criado y Rojas (2015) coincidían con estudios que se habían llevado a cabo anteriormente y que ya apuntaban a que los objetivos, en términos de participación y de gobierno abierto, no se estaban cumpliendo. En 2013, Yi, Oh y Kim (2013) llegaron a la conclusión de que la Administración pública estaba en una fase inicial, en cuanto a la implantación de redes sociales. Un año más tarde, en 2014, Mickoleit y Gascó, en estudios diferentes, identificaron carencias en la dimensión estratégica del uso de las redes sociales en la Administración pública. Y en 2020, con este trabajo de los tres ayuntamientos catalanes, no se dibuja un escenario demasiado distinto. Ocurre lo mismo en otro estudio titulado: "La participación en la Administración General del Estado a través de los medios digitales”, de Rafael Rubio y Ana Ibarz (2019), que considera que todavía queda un largo camino por recorrer para fomentar e impulsar la participación de la sociedad en los procesos, decisiones y actividades públicas.

\subsection{Comunicación de las instituciones públicas vs. comunicación política}

Los distintos autores recogidos en el apartado del marco teórico sostienen que, en muchas ocasiones, se hace difícil dibujar una línea fija que sirva de guía para separar lo que es comunicación de las instituciones públicas de lo que es comunicación política (Aira-Foix et al. 2019; Canel-Crespo, 2018; Escalona-Nicolás, 2015). Los resultados de los tres ayuntamientos analizados confirman también esta teoría.

Existen distintas publicaciones en las que se hace evidente que la información que se ofrece desde la Administración local es de servicio público, netamente objetiva y neutra, y que difunde únicamente la actividad del Ayuntamiento en cuestión, lo que se correspondería al 100\% con la comunicación de las instituciones públicas. 
En el Ayuntamiento de Barcelona, son claros los ejemplos en este sentido: desde la información sobre un nuevo espacio de duelo neonatal que se ha abierto en el cementerio, los resultados de la encuesta sobre Juventud, o la noticia sobre una nueva aplicación que permite al ciudadano hacer trámites en línea. Todos estos casos, pues, se traducen en comunicaciones explícitamente informativas.

Al otro lado del abanico encontramos publicaciones que no ofrecen ningún tipo de información de servicio público y que solamente hacen referencia a lo que hace el alcalde o la alcaldesa. Sin embargo, también hallamos publicaciones que mezclan parte de información de servicio público y de actividad del Ayuntamiento con parte de presencia de políticos y logros del gobierno, todo ello con tintes de contenido político/ideológico, a veces introducidos con brocha gorda, a veces de forma más sutil. Se trata de publicaciones que siguen —en más o menos medida - la receta de la campaña permanente de Sidney Blumenthal (1980), que explicita el fenómeno en el que gobernar se vuelve una campaña perpetua durante los cuatro años formales de legislatura en los que los dirigentes mantienen el proceso de venta del producto político de manera sostenida.

En todas las publicaciones analizadas hay información de servicio público, pero se adivina también una voluntad política de poner al líder como autor del logro y protagonista de la noticia.

\subsection{La ciudadanía a través del like}

La Administración pública tiene el deber de informar a los ciudadanos de todo aquello que les afecta y las redes sociales se dibujan como una herramienta facilitadora de esa función: que llegue el mensaje a la ciudadanía de una forma directa a su ordenador, tableta o móvil.

Como se puede ver en los resultados de este estudio, los tres ayuntamientos analizados consiguen un alto porcentaje de publicaciones que contienen información de servicio público. Sin embargo, las publicaciones que cosechan un porcentaje más alto de likes no coinciden, en su mayoría, con las que se podrían considerar informaciones que explican el desarrollo de políticas municipales que afectan al día a día de la ciudadanía, como podrían ser los proyectos que se aprueban en un pleno municipal.

En el TW del Ayuntamiento de Barcelona, la publicación que genera, de largo, más adhesión es un hilo del 26 de octubre de 2020 con fotografías profesionales de animales del Atlas de la Biodiversidad de la Ciudad (81 likes y 17 comparticiones). También propicia adhesión el tuit del 4 de noviembre que promueve la acogida de perros con un vídeo con "canes de ojos solitarios" (60 likes y 56 comparticiones). Son números muy por encima de la media (4). Otro ejemplo lo encontramos en el IG de Manresa, donde las fotos que más likes consiguen son la de la fachada del 
Ayuntamiento iluminada de color rosa y azul con motivo del Día del Duelo Gestacional (379 likes) y la del Anillo Verde, que consigue 394 likes. Por el contrario, la crónica del pleno (15 de octubre en FB) suma solamente 4 likes.

También se disparan las reacciones con aquellas noticias que generan un rechazo mayoritario por parte de la población, como el anuncio de la puesta en marcha de la Administración electrónica en Sabadell (9 de octubre FB e IG) o, por el contrario, las que consiguen conectar de pleno con la ciudadanía de manera positiva, como el anuncio del Ayuntamiento de Manresa de poner a disposición de la restauración local un servicio municipal de reparto de comida a domicilio para que puedan contrarrestar el cierre de sus locales.

La reflexión en este apartado de las conclusiones nos lleva a plantear más cuestiones para futuras investigaciones que versan sobre lo que busca la ciudadanía en las redes sociales, sobre el uso que hacen de ellas (con una finalidad informativa o, simplemente, lúdica), o sobre qué debe hacer la Administración pública para generar más atención hacia las políticas que afectan directamente a los ciudadanos y hacia la mejora del conocimiento de la propia institución.

\section{Referencias}

Agostino, Deborah (2013). Using social media to engage citizens: A study of Italian municipalities. En: Public Relations Review, Vol. 39, $\mathrm{n}^{\circ}$ 3. Nueva York: Elsevier, 232-234. DOI: http://dx.doi.org/10.1016/j.pubrev.2013.02.009

Aira-Foix, Toni; Curto-Gordo, Víctor; Escalona-Nicolás, Núria y Rom-Rodríguez, Josep (2019). Comunicació política i d’institucions públiques. Barcelona: Editorial UOC.

Baldwin-Philippi, Jessica (2015). Using technology, building democracy. Nueva York: Oxford University Press.

Bertot, John C.; Jaeger, Paul T. y Hansen, Derek (2012a) The impact of polices on government social media usage: Issues, challenges, and recommendations. En: Government Information Quarterly, Vol..29, n¹. Nueva York: JAI, 30-40. DOI: http://dx.doi.org/10.1016/j.giq.2011.04.004

Bertot, John C.; Jaeger, Paul T. y Grimes, Justin M. (2012b). Promoting transparency and accountability through ICTs, social media, and collaborative e-government. En: Transforming Government People Process and Policy, Vol. 6, n ${ }^{0} 1$. Bradford: Emerald, 78-91. DOI: http://dx.doi.org/10.1108/17506161211214831

Blumenthal, Sidney (1980). The Permanent Campaign. Boston: Beacon Press.

BOE (2005). Ley 29/2005, de 29 de diciembre, de Publicidad y Comunicación Institucional. 
Bonsón-Ponte, Enrique; Royo, Sonia y Ratkai, Melinda (2013). Analysis of European municipalities 'Facebook channels activity and citizens' engagement. Conference: AECA XVII Congress. Pamplona. Spain. http://www.aeca1.org/pub/on_line/comunicaciones_xviicongresoaeca/cd/84g.pdf

Boyd, Danah y Ellison, Nicole B. (2007) Social network sites: Definition, history, and scholarship. En: Journal of Computer-Mediated Communication, Vol. 13, $\mathrm{n}^{\mathrm{o}}$ 1. Oxford: Oxford University Press, a. 11. DOI: http://dx.doi.org/10.111 1/j.10836101.2007.00393.x

Campillo-Alhama, Conchi (2010). Comunicación pública y administración municipal. Una propuesta de modelo estructural. En: Pensar la Publicidad, vol.4, $\mathrm{n}^{0} 1$. Madrid: Universidad Complutense de Madrid, 45-62.

Canel-Crespo, María-José (2018). La comunicación de la administración pública. Para gobernar con la sociedad. México: Fondo de Cultura Económica,

Casero-Ripollés, Andreu (2018). Research on political information and social media: Key points and challenges for the future. En: El profesional de la información, Vol. 27, $\mathrm{n}^{\circ}$ 5. Barcelona: EPI, 964-974. DOI: https://doi.org/10.3145/epi.2018. sep.01

Chaves-Montero, Alfonso (2017). Comunicación Política y Redes Sociales. Sevilla: Ediciones Egregius.

Chun, Soon A.; Shulman, Stuart; Sandoval, Rodrigo y Hovy, Eduard (2010). Government 2.0. Making Connections between Citizens, Data and Government. Information Polity. En: The International Journal of Government \& Democracy in the Information Age, $\mathrm{n}^{0}$ 15. Ámsterdam: IOS Press, 1-9. DOI: http://dx.doi. org/10.3233/IP-2010-0205

Cervi, Laura (2020a). Veni, vidi, Facebooked-live: análisis del éxito de Matteo Salvini en Facebook. En: Revista CIDOB d'Afers Internacionals, n ${ }^{\circ}$ 124. Barcelona: CIDOB, 99-122. DOI: https://doi.org/10.24241/rcai.2020.124.1.99

Cervi, Laura (2020b). Exclusionary Populism and Islamophobia: A comparative analysis of Italy and Spain. En: Religions, Vol. 11. Basilea: MDPI, 516. DOI: https:// doi.org/10.3390/rel11100516

Cervi, Laura y Roca-Trenchs, Núria (2017a). Towards an Americanization of political campaigns? The use of Facebook and Twitter for campaigning in Spain, USA and Norway. En: Anàlisi, nº56. Barcelona: UAB, 87-100. DOI: https://doi. org/10.5565/rev/analisi.3072

Cervi, Laura y Roca-Trenchs, Núria (2017b). La modernización de la campaña electoral para las elecciones generales de España en 2015. ¿Hacia la americanización?. En: Comunicación y hombre, $\mathrm{n}^{\mathrm{o}}$ 13. Madrid: Universidad Francisco de Vitoria, 133-150. 
Criado, Juan-Ignacio (2013). “Open Government, Social Media y Sector Público”. En: R. Cotarelo (ed.). Ciberpolítica. Las Nuevas Formas de Acción Política. Valencia: Tirant lo Blanc.

Criado, Juan-Ignacio y Villodre, Julián (2021). Delivering public services through social media in European local governments. An interpretative framework using semantic algorithms. En: Local Government Studies, Vol. 47, n ${ }^{0} 2$. Londres: Taylor \& Francis, 253-275. DOI: https://doi.org/10.1080/03003930.2020.1729750

Criado, Juan-Ignacio y Pastor, Vicente; Villodre, Julián (2018). Measuring Social Media Diffusion in Local Governments from a Longitudinal Perspective: Adoption, Barriers, and Perceptions. En: Sobaci, M. H. y Hatipoglu, I. (Eds.) (2018). SubNational Democracy and Politics Through Social Media. ueva York: Springer. DOI: https://doi.org/10.1007/978-3-319-73386-9

Criado, Juan-Ignacio y Villodre, Julián (2018). Comunicando Datos Masivos Del Sector Público Local En Redes Sociales. Análisis De Sentimiento En Twitter. En: Profesional de la Información, Vol. 27, $\mathrm{n}^{0} 3$. Barcelona: EPI: 624-32. DOI: https:// doi.org/10.3145/epi.2018.may.14

Criado, Juan-Ignacio; Pastor, Vicente y Villodre, Julián (2017). Redes sociales digitales en los ayuntamientos españoles. En: Laboratorio Iberoamericano de Gobierno para la Innovación Pública. ${ }^{0} 2$. Madrid: Universidad Autónoma de Madrid. Criado, Juan-Ignacio y Rojas-Martín, Francisco (2012). Strategies and Realities of Social Media Diffusion in the Public Sector. Evidence from the Regional Government in Spain. Documento presentado en la reunion anual del European Group of Public Administration. Bergen, Noruega.

Criado, Juan-Ignacio y Rojas-Martín, Francisco (2013). Social Media and Public Administration in Spain. A Comparative Analysis of the Regional Level of Government. En: J. R. Gil-García (Ed.), e-Government success around the world: cases, empirical studies, and practical recommendations.. Hershey: IGI Global.

Criado, Juan-Ignacio y Rojas-Martín, Francisco (2015). El impacto de las redes sociales en las administraciones locales. Mitos y realidades en el caso español. En: IDP. Revista de Internet, Derecho y Política, ${ }^{\circ} 20$. Barcelona: UOC, 25-42.

Dimitrova, Daniela V. y Matthes, Jörg (2018). Social media in political campaigning around the world: Theoretical andmethodological challenges. En: Journalism \& mass communication quarterly, Vol. 95, $\mathrm{n}^{0}$ 2. Londres: Sage, 333-342. https:// doi.org/10.1177/1077699018770437

Ellison, Nick y Hardey, Michael (2014). Social Media and Local Government: Citizenship, Consumption and Democracy. En: Local Government Studies, Vol. 40, $\mathrm{n}^{\circ}$ 1. Londres: Taylor \& Francis, 21-40. DOI: http://dx.doi.org/10.1080/03 003930.2013 .799066 
Escalona-Nicolás, Núria (2015). Comunicación de instituciones públicas. Barcelona: Editorial UOC.

Fernández-Falero, María-Rosario; Trabadela-Robles, Javier; Garcés-Botacio, Indhira y Ruano-López, Soledad (2017). Comunicación política De Los Ayuntamientos a través De Sus Webs. Caso De Extremadura. En: Profesional De La Información, Vol. 26, nº3. Barcelona: EPI, 404-11. DOI: https://doi.org/10.3145/epi.2017.may.06 Gascó, Mila (2014). Xarxes socials, transparència i govern obert a Catalunya. Barcelona: ESADE / Universitat Ramon Llull.

Graells-Costa, Jordi y Ramilo-Araujo, Mentxu (2013). Ciudadanía y administraciones en red: la Administración Pública ante la nueva sociedad en red. Oleiros: Netbiblo.

Graham, Melissa y Avery, Elisabeth (2013). Government Public Relations and Social Media: An Analysis of the Perceptions and Trends of Social Media Use at the Local Government Level. En: Public Relations Journal, Vol. 7, n ${ }^{0}$ 4. Nueva York: PSA, 1-21.

Guitérrez-Rubí, Antonio (2014). Campaña y comunicación permanente. Consultado el 20/01/2022 en https://www.gutierrez-rubi.es/2014/10/27/campana-y-comunicacion-permanente/

Hong, Hyehyun (2013). Government websites and social media's influence on government-public relationships. En: Public Relations Review, Vol.39, $\mathrm{n}^{\circ} 4$. Nueva York: Elsevier, 346-356. DOI: http://dx.doi.org/10.1016/j.pubrev.2013.07.007

IAB Spain (2019). Las redes sociales se consolidan como canal de influencia e información. Consultado el 20/01/2022 en https://iabspain.es/etiqueta/estudioanual-de-redes-sociales/

INE, Instituto Nacional de Estadística (2020). Población que usa internet. Tres últimos meses de 2019. Tipo de actividades realizadas. Consultado el 20/01/2022 en https://www.ine.es/ss/Satellite?L=es_ES\&c=INESeccion_C\&cid=125992552878 $2 \& p=1254735110672 \&$ pagename=ProductosYServicios\%2FPYSLayout

Institut d’Estadística de Catalunya (2019). Dades de població. Consultado el 20/01/2022 en https://www.idescat.cat/tema/xifpo

Medaglia, Rony y Zheng, Lei (2017). Mapping government social media research and moving it forward: A framework and a research agenda. En: Government Information Quarterly, Vol. 34, nº3. Nueva York: JAI, 496-510. DOI: https://doi. org/10.1016/j.giq.2017.06.001

Meijer, Albert y Thaens, Marcel (2013). Social media strategies: Understanding the differences between North American police departments. En: Government Information Quarterly, Vol. 30, $\mathrm{n}^{\mathrm{o}}$ 4. Nueva York: JAI, 343-350. DOI: http://dx.doi. org/10.1016/j.giq.2013.05.023 
Mickoleit, Arthur (2014). Social Media Use by Governments: A Policy Primer to Discuss Trends, Identify Policy Opportunities and Guide Decision Makers. En: OECD Working Papers on Public Governance. $\mathrm{n}^{0} 26$. Paris: OECD Publishing. DOI: http://dx.doi.org/10.1787/5jxrcmghmk0s-en

Mossberg, Karen; Wu, Yonghong y Crawford, Jared (2013). Connecting citizens and local governments? Social media and interactivity in major U.S. cities. En: Government Information Quarterly, Vol. 30, $\mathrm{n}^{0} 4$. Nueva York: JAI 351-358. DOI: http://dx.doi.org/10.1016/j.giq.2013.05.016

Papacharissi, Zizi. (2010). A Private Sphere: Democracy in a Digital Age. Cambridge: Polity.

Rivero-Hernández, Magda (2017). La comunicación en las instituciones de la Administración Pública. Consultado el 20 de enero de 2022 en https://www.researchgate.net/publication/320871128

Rubio-Núñez, Rafael y Ibarz-Moret, Ana (2019). La participación en la Administración General del Estado a través de los medios digitales. Madrid: Centro de Estudios Políticos y Constitucionales. Subsecretaria del Ministerio de Presidencia.

Song, Changsoo y Lee, Jooho (2015). Citizens' Use of Social Media in Government, Perceived Transparency, and Trust in Government. En: Public Performance \& Manager Review, Vol. 39. Armon: Sharpe, 430-453. DOI: https://doi.org/10.1 080/15309576.2015.1108798

Vasko; Vidar y Trilling, Damian (2019). A permanent campaign? Tweeting differences among members of Congress between campaign and routine periods. En: Journal of information technology \& politics, Vol. 16, $\mathrm{n}^{\circ} 4$. Binghartom: Haworth Press, 342-359.DOI: https://doi.org/10.1080/19331681.2019.1657046

Vergeer, Maurice; Hermans, Liesbeth y Cunha, Carlos (2013). Web campaigning in the 2009 European Parliament elections: A cross-national comparative analysis. En: New Media and Society, Vol. 15, $\mathrm{n}^{\circ}$ 1. Londres: Sage 128-148. DOI: https:// doi.org/10.1177/1461444812457337

Wukich, Clayton (2021). Government Social Media Engagement Strategies and $\mathrm{Pu}-$ blic Roles. En: Public Performance \& Management Review, Vol. 44, ${ }^{\circ} 1$. Armonk: Sharpe, 187-215. DOI: https://doi.org/10.1080/15309576.2020.1851266

Yi, Myongho; Oh, Sam-Gyun y Kim, Sunghun (2013). Comparison of social media use for the U.S. and the Korean governments. Government Information Quarterly, Vol. 30, $\mathrm{n}^{\mathrm{o}}$ 3. Nueva York: JAI, 310-317. DOI: http://dx.doi.org/10.1016/j. giq.2013.01.004

Zavattaro, S. M., French, P. E. y Mohanty, S. D. (2015). A sentiment analysis of U.S. local government tweets: The connection between tone and citizen involvement. En: Government Information Quarterly, Vol. 32. Nueva York: JAI, 333-341. DOI: https://doi.org/10.1016/j.giq.2015.03.003 